Anaesthesist 2011 $\cdot 60: 201-202$ DOI 10.1007/s00101-011-1876-1 Online publiziert: 10. März 2011 (c) Springer-Verlag 2011

\section{T. Volk $\cdot$ R. Breitkreutz}

Klinik für Anästhesiologie, Intensivmedizin und Schmerztherapie, Universitätsklinikum des Saarlandes, Homburg

\title{
Kompetenzentwicklung in der Sonographie für unser Fach
}

\author{
Epluribus unum
}

Wir stehen am Beginn einer neuen Zeit für Ultraschallverfahren in unserem Fach. Nachdem die Vorteile bei der Durchführung von regionalen Anästhesie- und Analgesieverfahren offenkundig sind, locken weitere Anwendungen in der Akut- und Intensivmedizin.

Man stelle sich vor: Es ist Samstagnacht, und Ihr Patient entwickelt einen unklaren Schockzustand. Aus dem klinischen Kontext heraus haben Sie den Verdacht auf eine Lungenembolie. Der Radiologe und der Kardiologe sind zu Hause; der Patient ist für einen Transport zur Computertomographie zu ,instabil“. Wäre es jetzt nicht angemessen oder sogar zu fordern, einen Blick auf das Herz zu wagen und im Fall der Identifikation einer akuten Rechtsherzbelastung eine zielorientierte Therapie einzuleiten? Die Antwort auf diese Frage lautet „Ja“: Die Leitlinie der Europäischen Gesellschaft für Kardiologie (ESC) zur Diagnostik und Therapie der Lungenembolie aus dem Jahr 2008 empfiehlt ganz eindeutig, eine Echokardiographie unabhängig von der fachärztlichen Spezialisierung vorzunehmen, um nach Zeichen der akuten Rechtsherzbelastung zu fahnden [1].

Intensiv- und Akutmediziner können von einer bettseitigen Diagnostik besonders profitieren. Vielen wird die Situation vertraut sein, dass erst nach einer CTUntersuchung die Ursache eines „Weaning"-Versagens auf Pleuraergüsse zurückzuführen ist. In einer vergleichenden
Untersuchung fanden Lichtenstein et al. [2], dass eine einfache Ultraschalluntersuchung bei Patienten mit ,acute respiratory distress syndrome" (ARDS) sowohl der Auskultation als auch einer Thoraxröntgenaufnahme in der diagnostischen Sicherheit überlegen ist. Im damaligen Editorial wurde bereits provozierend die Frage gestellt, ob wir das Stethoskop nicht bald an den Nagel hängen können [3].

Es gibt mittlerweile brillante und einfache Methoden, die aufzeigen, wie wertvoll Ultraschalldiagnostik in der Intensiv- und Notfallmedizin sein kann: So lässt sich ein kardial-bedingtes Lungenödem bereits im subklinischen Stadium mit Ultraschall identifizieren [4], das Ansprechen auf Volumentherapie bei Sepsis durch Beurteilung der Kollapsibilität der V. cava superior vorhersagen [5] oder gar ein okkulter Pneumothorax diagnostizieren [6]. Es handelt sich für Geübte meist um Blickdiagnosen, oder es sind nur wenig aufwendige Messungen notwendig, um eine Diagnose zu stellen oder auszuschließen.

Gut belegt ist zudem, dass die Verwendung von Ultraschall bei der Anlage zentraler vaskulärer Zugänge die Versagerrate, die Risiken für Komplikationen und die Zahl der Punktionsversuche gegenüber einer auf Landmarken gestützten Punktion reduzieren kann $[7,8,9]$. Nach einer Kosten-Nutzen-Analyse kam das National Institute for Health and Clinical Excellence in Großbritannien bereits 2002 zu der Empfehlung, dass zentrale Gefäß- punktionen ultraschallkontrolliert erfolgen sollen: Ein solches Vorgehen scheint nicht nur sicherer zu sein, sondern spart auch 2 GBP/Patient (www.nice.org.uk/). Die technologische Entwicklung von Geräten, die bereits die Größe von „smartphones" erreicht haben, lässt vermuten, dass die Anschaffungskosten in den kommenden Jahren noch einmal deutlich reduziert werden.

Der Leitartikel dieser Ausgabe von Maecken et al. [10] greift die zunehmende Bedeutung des Ultraschalls für unser Fachgebiet auf und liefert grundlegende Einblicke in die Thematik. Der interessierte und lernwillige Leser steht allerdings vor dem Dilemma, dass in Deutschland für das Erlernen der Ultraschallbildgebung im Umfeld von Intensiv- und Notfallmedizin noch kein einheitliches Curriculum existiert und auch bei den meisten potenziellen Anwendern nur limitierte Vorstellungen über die Möglichkeiten der Technik vorliegen. International sieht dies bereits deutlich anders aus. So gibt es neben dem Curriculum der „European Federation of Societies for Ultrasound in Medicine and Biology" (EFSUMB) weitere Initiativen von führenden Fachgesellschaften oder Expertengruppen, die Lerninhalte aus Sicht des „critical care physician" definieren $[11,12,13,14,15]$. Dabei ist immer klar: Die Brücke für eine sinnvolle Anwendung der Sonographie in der Intensiv- und Notfallmedizin ist eine gute Ausbildung in der Technik. 
Im Gegensatz zu vielen anderen klinischen Fächern gab es in der Anästhesiologie und Intensivmedizin - mit Ausnahme des mittlerweile gut etablierten Curriculums für transösophageale Echokardiographie - lange Zeit kein weiterführendes (Ausbildungs-)Konzept. Dies soll sich in Kürze ändern: Ein entsprechendes Curriculum wird derzeit durch die Deutsche Gesellschaft für Anästhesiologie und Intensivmedizin (DGAI) zusammen mit zahlreichen Experten erarbeitet.

Dieser Schritt ist sehr zu begrüßen, da der Weg der orientierenden Diagnostik mit dem geübten Auge („eye-balling“) auf einer niedrigen Ausbildungsstufe natürlich fehleranfällig ist und bleibt. So kann im eingangs gewählten Beispiel der Lungenembolie selbst für den Geübten die Differenzialdiagnose zum akuten Cor pulmonale bei vorbestehender Rechtsherzbelastung ohne Lungenembolie [z. B. bei „chronic obstructive pulmonary disease" (COPD) oder ARDS] trotz technisch korrekter Quantifizierung der akuten Rechtsherzbelastung eine Herausforderung darstellen.

Freuen können wir uns jedoch sicher darauf, dass zukünftig eine Vielzahl konkreter Probleme und Fragestellungen immer häufiger ausschließlich sonographisch lösbar sein wird. Die Beispiele hierfür sind mannigfaltig und umfassen die Diagnosen einer Perikardtamponade, einer akuten akalkulösen Cholezystitis oder das Vorhandensein von Peristaltik oder Zwerchfellbewegungen ebenso wie die Abschätzung des Füllungszustands des Magens oder die Quantifizierung der Harnblasenfüllung.

Vielleicht werden wir jedoch dabei auch lernen müssen, dass im Bereich der Intensivmedizin eine -gegenüber anderen Szenarien - noch systematischere und standardisiertere Art der Untersuchung erforderlich ist, weil Pathophysiologie und Dynamik lebensbedrohlicher Erkrankungen so komplex sind, dass mehrere Spezialisten mit ihren Erfahrungen in der Bildgebung miteinander kooperieren und hierbei eine einheitliche „Sprache“ sprechen müssen.

Insgesamt ist es also allerhöchste Zeit für unser Fach, dass wir uns nicht nur mit dem „Einsammeln“ zahlreicher Spezialkompetenzen in der Sonographie be- schäftigen, sondern unseren Patienten offensichtliche Vorteile für mehr Sicherheit schnellstmöglich zukommen lassen. Wir können gespannt sein, wie schnell die neuen Möglichkeiten einer „Pointof-care-"Diagnostik in Echtzeit [16] auch hierzulande Einzug halten werden. Wie auch immer die rasante Entwicklung weitergehen wird; sie wird uns - bei allen Unwägbarkeiten und mancherlei Bedenken im Detail - bereichern getreu dem Motto von Georg Christoph Lichtenberg:
- „Ich weiß nicht, ob es besser wird, wenn es anders wird. Aber es muss anders werden, wenn es besser werden soll." ([17])

\section{Korrespondenzadresse \\ Prof. Dr. T. Volk \\ Klinik für Anästhesiologie, Intensiv- medizin und Schmerztherapie, Uni- versitätsklinikum des Saarlandes Gebäude 57, 66421 Homburg \\ Thomas.Volk@uniklinikum-saarland.de}

\section{Literatur}

1. Torbicki A, Perrier A, Konstantinides S et al; ESC Committee for Practice Guidelines (CPG) (2008) Guidelines on the diagnosis and management of acute pulmonary embolism: the Task Force for the Diagnosis and Management of Acute Pulmonary Embolism of the European Society of Cardiology (ESC). Eur Heart J 29:2276-2315

2. Lichtenstein D, Goldstein I, Mourgeon E et al (2004) Comparative diagnostic performances of auscultation, chest radiography, and lung ultrasonography in acute respiratory distress syndrome. Anesthesiology 100:9-15

3. Hubmayr RD (2004) The times are a-changin': should we hang up the stethoscope? Anesthesiology 100:1-2

4. Gargani L, Frassi F, Soldati G et al (2008) Ultrasound lung comets for the differential diagnosis of acute cardiogenic dyspnoea: a comparison with natriuretic peptides. Eur J Heart Fail 10:70-77

5. Vieillard-Baron A, Charron C, Chergui K et al (2006) Bedside echocardiographic evaluation of hemodynamics in sepsis: is a qualitative evaluation sufficient? Intensive Care Med 32:1547-1552

6. Lichtenstein DA, Mezière G, Lascols N et al (2005) Ultrasound diagnosis of occult pneumothorax. Crit Care Med 33:1231-1238

7. Randolph AG, Cook DJ, Gonzales CA, Pribble CG (1996) Ultrasound guidance for placement of central venous catheters: a meta-analysis of the literature. Crit Care Med 24:2053-2058

8. Hind D, Calvert N, McWilliams R et al (2003) Ultrasonic locating devices for central venous cannulation: meta-analysis. BMJ 327:361-8
9. Scheiermann P, Seeger FH, Breitkreutz R (2010) UItrasound-guided central venous access in adults and children: procedure and pathological findings. Anaesthesist 59:53-61

10. Maecken T, Zinke H, Zenz M, Grau T (2001) In welcher Weise sollte ein Anästhesist sonographieren können? Diagnostischer Einsatz des Ultraschalls in der Akut- und Intensivmedizin. Anaesthesist 60:203-213

11. Mayo PH, Beaulieu Y, Doelken P et al (2009) American College of Chest Physicians/La Société de Réanimation de Langue Française statement on competence in critical care ultrasonography. Chest 135:1050-1060

12. Frenkel H, Levitov A, Desai H et al (2011) Guidelines for the appropriate use of bedside general and cardiac ultrasonography by the intensivist in the evaluation of critically ill patients. Crit Care Med (im Druck)

13. Cholley B et al. (2011) International expert statement on training standards for critical care ultrasonography. Intensive Care Med (im Druck)

14. Price S, Via G, Sloth E et al; World Interactive Network Focused On Critical UltraSound ECHO-ICU Group (2008) Echocardiography practice, training and accreditation in the intensive care: document for the World Interactive Network Focused on Critical Ultrasound (WINFOCUS). Cardiovasc Ultrasound 6:49

15. Neri L, Storti E, Lichtenstein D (2007) Toward an ultrasound curriculum for critical care medicine. Crit Care Med 35(5 Suppl):290-304

16. Moore CL, Copal JA (2011) Current concepts: point of care ultrasonography. N Engl J Med 364:749757

17. Lichtenberg GC (1984) Sudelbücher. Insel Taschenbuch. Suhrkamp, Berlin 